\title{
Higher caseload improves cervical cancer survival in patients treated with brachytherapy
}

\author{
Moon-Sing Lee ${ }^{1,4 \dagger}$, Shiang-Jiun Tsai ${ }^{1 \dagger}$, Ching-Chih Lee ${ }^{2,4}$, Yu-Chieh Sü ${ }^{3,4}$, Wen-Yen Chiou, ${ }^{1,4}$, Hon-Yi Lin ${ }^{1,4^{*}}$ \\ and Shih-Kai Hung ${ }^{1,4^{*}}$
}

\begin{abstract}
Objectives: Increased caseload has been associated with better patient outcomes in many areas of health care, including high-risk surgery and cancer treatment. However, such a positive volume vs. outcome relationship has not yet been validated for cervical cancer brachytherapy. The purpose of this study was to examine the relationship between physician caseload and survival rates in cervical cancer treated with brachytherapy using population-based data.

Methods: Between 2005 and 2010, a total of 818 patients were identified using the Taiwan National Health Insurance Research Database. Multivariate analysis using a Cox proportional hazards model and propensity scores was used to assess the relationship between 5-year survival rates and physician caseloads.

Results: As the caseload of individual physicians increased, unadjusted 5-year survival rates increased $(P=0.005)$. Using a Cox proportional hazard model, patients treated by high-volume physicians had better survival rates $(P=0.03)$, after adjusting for comorbidities, hospital type, and treatment modality. When analyzed by propensity score, the adjusted 5-year survival rate differed significantly between patients treated by high/medium-volume physicians vs. patients treated by low/medium-volume physicians ( $60 \%$ vs. $54 \%$, respectively; $P=0.04$ ).
\end{abstract}

Conclusions: Provider caseload affected survival rates in cervical cancer patients treated with brachytherapy. Both Cox proportional hazard model analysis and propensity scores showed association between high/medium volume physicians and improved survival.

\section{Introduction}

Cervical cancer remains the most important cause of cancer death in women from Taiwan with an ageadjusted incidence of 26.2 per one hundred thousand women $[1,2]$. The combination of chemotherapy administered concurrently with radiotherapy shows survival benefit in patients with bulky and locally advanced cervical cancer [3]. However, dose is related to both local control of tumor growth and overall survival. The risk of tissue toxicity currently limits the external radiation dose that can be safely delivered [4]. Thus, brachytherapy is often combined with external beam radiotherapy in

\footnotetext{
* Correspondence: DOC31221@ndmctsgh.edu.tw;

oncology158@yahoo.com.tw

${ }^{\dagger}$ Equal contributors

'Department of Radiation Oncology, Buddhist Dalin Tzu Chi Hospital, 2, Ming Sheng Road, Dalin, Chiayi, Taiwan

${ }^{4}$ School of Medicine, Tzu Chi University, Hualien, Taiwan

Full list of author information is available at the end of the article
}

cervical cancer patients to prevent damage to surrounding normal tissues.

Brachytherapy is a technically demanding process. The "practice makes perfect" hypothesis may be valid for such a procedure, in that increased experience improves patient outcomes. The association between increased caseload and improved patient outcomes has been reported for both high-risk surgery and cancer treatment $[1,2]$. Positive correlations between improved treatment outcomes and increased caseload volume have been documented for nasopharyngeal cancer, breast cancer, oral cancer, and esophageal cancer [2,5-7]. However, such a positive volume-outcome relationship has not been established for cervical cancer brachytherapy. The purpose of this study was to examine the relationship between physician caseload and survival rates in cervical cancer patients treated with brachytherapy, using population-based data.

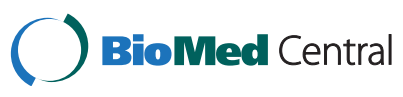

(C) 2014 Lee et al.; licensee BioMed Central Ltd. This is an Open Access article distributed under the terms of the Creative Commons Attribution License (http://creativecommons.org/licenses/by/2.0), which permits unrestricted use, distribution, and reproduction in any medium, provided the original work is properly credited. The Creative Commons Public Domain Dedication waiver (http://creativecommons.org/publicdomain/zero/1.0/) applies to the data made available in this article unless otherwise stated. 


\section{Materials and methods Ethics statement}

The study protocol was approved by the Buddhist Dalin Tzu Chi General Hospital Institutional Review Boards. The institutional review board waived the need for written informed consent from the participants because the data analyzed consisted of anonymous secondary data released to the public for research.

\section{Patients and study design}

Between 2005 to 2010, data from the National Health Insurance (NHI) Research Database was used in this study. This data contained all covered medical benefit claims for over 23 million people in Taiwan (approximately 97 percent of the island's population). All patients with cervical cancer (as defined by International Classification of Disease, Ninth Revision, Clinical Modification codes 180) were included who received radiotherapy or chemoradiotherapy between 2005 and 2007. Patients were excluded who had unclear treatment modalities or incomplete physician data. Finally, 818 patients, treated by 93 radiation oncologists during this 5-year period, were included in our analysis.

Physicians were further stratified by their total patient volumes (using the unique physician identifiers in this database) and by their caseload of cervical cancer patients. The volume category cutoff points (high, medium, and low) were determined by sorting the 818 patients into three groups (1-11 cases $=$ low caseload), 12-40 cases $=$ medium caseload, and $\geqq 41$ cases $=$ high caseload), as previously described $[5,8]$. The volume category cutoff points were determined by sorting the sample into 3

Table 1 Patients characteristics according to caseload $(\mathbf{n}=\mathbf{8 1 8})$

\begin{tabular}{|c|c|c|c|c|}
\hline \multirow{4}{*}{ Variable } & \multicolumn{3}{|c|}{ Cervical cancer caseload group } & \multirow{4}{*}{$P$-value } \\
\hline & Low & Medium & High & \\
\hline & $(1-11)$ & $(12-40)$ & $(41-78)$ & \\
\hline & $(n=280)$ & $(n=262)$ & $(n=276)$ & \\
\hline Age & & & & $<0.001$ \\
\hline $25-44$ years & $23(8.2)$ & 29(11.1) & $40(14.5)$ & \\
\hline $45-54$ years & $53(18.9)$ & $57(21.8)$ & $74(26.8)$ & \\
\hline $55-64$ years & $47(16.8)$ & 33(12.6) & $58(21.0)$ & \\
\hline $65-74$ years & $76(27.1)$ & $68(26.0)$ & $60(21.7)$ & \\
\hline$\geqq 75$ years & $81(28.9)$ & $75(28.6)$ & $44(15.9)$ & \\
\hline Charlson comorbidity index score & & & & 0.009 \\
\hline 0 & 103(36.8) & 113(43.1) & 139(50.4) & \\
\hline $1-3$ & 109(38.9) & 78(29.8) & $80(29.0)$ & \\
\hline$\geqq 4$ & $68(24.3)$ & $71(27.1)$ & $57(20.7)$ & \\
\hline Treatment modality & & & & 0.001 \\
\hline Radiotherapy & 122(43.6) & $82(31.3)$ & $83(30.1)$ & \\
\hline Chemoradiotherapy & 158(56.4) & 180(68.7) & 193(69.9) & \\
\hline Geographic location & & & & $<0.001$ \\
\hline North & $98(35.0)$ & 108(41.2) & $57(20.7)$ & \\
\hline Central & $74(26.4)$ & 84(32.1) & 129(46.7) & \\
\hline Southern and Eastern & 108(38.6) & $70(26.7)$ & $90(32.6)$ & \\
\hline Enrollee category & & & & 0.89 \\
\hline EC 1-2 & $60(21.4)$ & $57(21.8)$ & $57(20.7)$ & \\
\hline EC 3 & 109(38.9) & 107(40.8) & $100(36.2)$ & \\
\hline EC 4 & $57(20.4)$ & $51(19.5)$ & $58(21.9)$ & \\
\hline Other & $54(19.3)$ & $47(17.9)$ & $61(22.1)$ & \\
\hline Urbanization & & & & 0.14 \\
\hline Urban & $66(23.6)$ & $84(32.1)$ & $73(26.4)$ & \\
\hline Suburban & 133(47.5) & 102(38.9) & 130(47.1) & \\
\hline Rural & $81(28.9)$ & $76(29.0)$ & $73(26.4)$ & \\
\hline
\end{tabular}

Values are given as number (percentage). 
approximately equal groups, so that each group would have approximately equal numbers of patients. These cervical cancer patients were then linked to death data extracted from the records covering the years between 1996 and 2010.

\section{Measurements}

The key dependent variable of interest was the 5-year survival rate. The key independent variables were the cervical cancer caseloads (low, medium, or high). Other physician characteristics included age ( $\leqq 40,41-50, \geqq 51$ years) and gender. Patient characteristics included age, geographic location, treatment modality, severity of disease, enrollee category (EC), and urbanization. The disease severity in each patient was assessed using the modified Charlson comorbidity index score, which has been widely used, in recent years, for risk adjustment in administrative claims data sets [9].

This study used EC as a proxy measure of socioeconomic status, which is an important prognostic factor in cancer patients [10,11]. Patients with cervical cancer were classified into four subgroups: EC 1 (civil servants, full-time, or regular paid personnel with a government affiliation), EC 2 (employees of privately owned institutions), EC 3 (self-employed individuals, other employees, and members of farmers' or fishermen's associations), EC 4 (veterans, low-income families, and substitute service draftees), and other [12]. In Taiwan, government affiliated workers have stable job occupation and fixed salary in every month than self-employed. On average, government affiliated workers' payroll-related amount for the health insurance was highest.

The hospitals were categorized by ownership (public, not-for-profit, or for-profit) and hospital type (medical center, regional hospital, or district hospital).

\section{Statistical analysis}

The SAS statistical package (version 9.2; SAS Institute, Inc., Cary, N.C.) and SPSS (version 21, SPSS Inc., Chicago, IL, USA) was used for data analysis. A two-sided $P$ value $<0.05$ was used to determine statistical significance.

The cumulative 5-year survival rates and the survival curves for each group were compared by the log-rank test. Survival was measured from the time of cervical cancer diagnosis to the time of death. Cox proportional regression model and survival analysis using propensity score stratification were used to compare outcomes between different groups based on caseload.

\section{Cox proportional hazards model}

The Cox proportional regression model was used to evaluate the effect of caseload on survival rate after
Table 2 Physician characteristics $(n=93)$

\begin{tabular}{lllll}
\hline \multirow{2}{*}{ Variable } & \multicolumn{3}{l}{ Physician caseload group } & \\
\cline { 2 - 4 } & $\begin{array}{l}\text { Low } \\
(\mathbf{1 - 1 1 )}\end{array}$ & $\begin{array}{l}\text { Medium } \\
\mathbf{( 1 2 - 4 0 )}\end{array}$ & $\begin{array}{l}\text { High } \\
\mathbf{( 4 1 - 7 8 )}\end{array}$ & \\
\hline $\begin{array}{l}\text { Total no. of physicians } \\
\text { Age (years) }\end{array}$ & 74 & 14 & 5 & \\
$\quad \begin{array}{l}\text { Mean } \pm \text { SD } \\
\text { Gender }\end{array}$ & $42 \pm 8$ & $41 \pm 6$ & $41 \pm 4$ & \\
$\quad \begin{array}{l}\text { Male } \\
\text { Female }\end{array}$ & $64(86)$ & $13(92)$ & $4(80)$ & \\
Caseload & $10(13)$ & $1(7)$ & $1(20)$ & \\
$\quad$ Mean \pm SD & $3 \pm 2$ & $18 \pm 7$ & $55 \pm 13$ & \\
\hline
\end{tabular}

Values are given as number (percentage).

Abbreviation: SD = standard deviation.

adjusting for hospital type, surgeon characteristics, and patient demographics.

\section{Propensity score}

Propensity analysis was used to reduce the effect of selection bias on our hypothesis, as described by Rosenbaum and Rubin [13-15]. Propensity score stratification replaced the many confounding factors that might be present in an observational study with such a variety of factors. To calculate the propensity score in this study, patient characteristics were entered into a logistic regression model that predicted selection for high/medium-volume surgeons. These patient characteristics included the year in which the patient was diagnosed, their age, gender, Charlson comorbidity index score, geographic area of residence, enrollee category, and treatment modality. The study population was then divided into five discrete strata based on propensity score. The effect of caseload assignment on 5 -year survival rates was analyzed within each quintile.

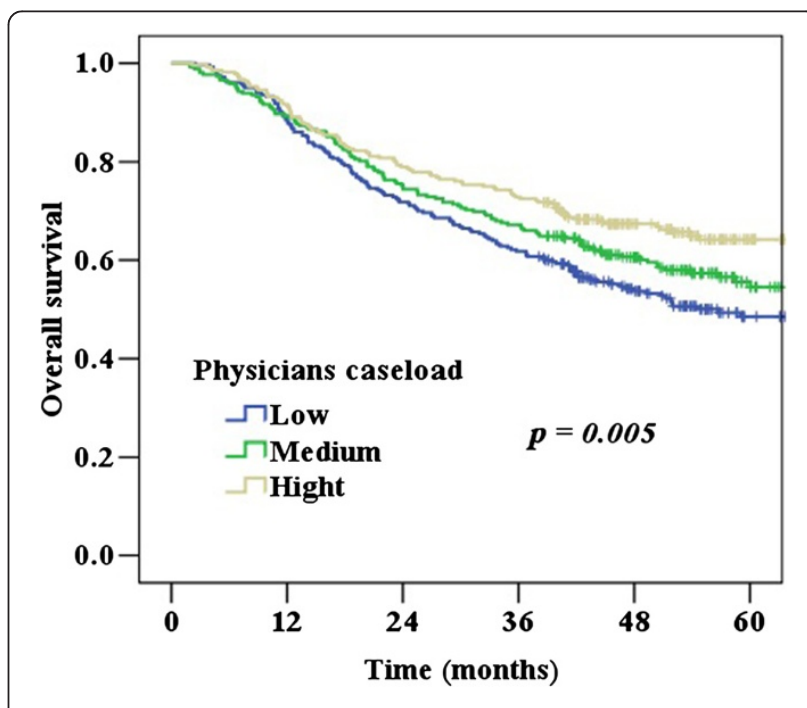

Figure 1 Cervical cancer survival rates by physician caseload. 
Table 3 Cervical cancer survival rate and adjusted hazard ratios by physician caseload groups and the characteristics of the patients and providers $(n=818)$

\begin{tabular}{llll}
\hline Variable & $\begin{array}{l}\text { Adjusted } \\
\text { hazard ratio }\end{array}$ & $95 \% \mathrm{Cl}$ & $P$-value \\
\hline
\end{tabular}

Physician characteristics

Physician volume

Low (1-11)

Medium (12-40)

High (41-78)

Physician age

$\leqq 40$ years

41-50 years

$\geqq 51$ years

Hospital characteristics

Hospital ownership

Public

Non-for-profit

For-profit

Hospital level

Medical center

Regional hospital

District hospital

Patient characteristics

Patient age

25-44 years

45-54 years

55-64 years

65-74 years

$\geqq 75$ years

Comorbidity index score

0

$1-3$

$\geqq 4$

Treatment modality

Chemoradiotherapy

Radiotherapy

Geographic location

North

Central

Southern and Eastern

Enrollee category

Other

EC 1-2

EC 3

EC 4 hazard ratio
0.90

0.69

(0.69-1.19) $\quad 0.49$

$(0.50-0.97) \quad 0.03$

0.94

0.88

(0.72-1.24) $\quad 0.70$

(0.56-1.36) $\quad 0.56$

(0.72-1.25) $\quad 0.74$

(0.69-1.40) $\quad 0.95$

(0.71-1.26) $\quad 0.70$

$(1.01-2.49) \quad 0.04$
Table 3 Cervical cancer survival rate and adjusted hazard ratios by physician caseload groups and the characteristics of the patients and providers $(n=818)$ (Continued)

\begin{tabular}{llll}
\hline $\begin{array}{l}\text { Urbanization } \\
\text { Urban }\end{array}$ & 1 & & \\
Suburban & 0.73 & $(0.56-0.95)$ & 0.02 \\
Rural & 0.66 & $(0.48-0.91)$ & 0.01 \\
\hline $95 \% \mathrm{Cl}, 95 \%$ confidence interval. & &
\end{tabular}

The Mantel-Haenszel odds ratio was calculated in addition to the Cochran-Mantel-Haenszel $\chi^{2}$ statistic.

\section{Results}

A total of 346 out of 818 patients (42\%) died after undergoing treatment between 2005 and 2007. A total of 93 radiation oncologists were included in the analysis. The characteristics of the physicians and patients are summarized in Tables 1 and 2. Patients in the low-volume physician group were more likely to undergo radiotherapy, reside in Southern and Eastern Taiwan, and have higher comorbidity score, than their counterparts in other groups. There were 74 radiation oncologists $(80 \%)$ in the low-volume group, 14 physicians $(15 \%)$ in the mediumvolume group, and five (5\%) physicians in the highvolume group. The mean age of all physicians was $41 \pm 6$ years. There was no significant difference among physicians who comprised these three caseload groups with regards to age $(P=0.90)$.

\section{Analysis using a Cox proportional hazards model}

The 5-year survival rates, by physician caseload group, are shown in Figure 1. The 5-year survival rates were $48 \%, 54 \%$, and $64 \%$ for low-, medium-, and high-volume surgeons, respectively $(P=0.005)$. Table 3 shows the adjusted hazard ratios (calculated using the Cox proportional hazards regression model) after adjusting for patient comorbidities, hospital type, and treatment modality. Physicians' age and 5-year survival have no association $(P>0.05)$. The hazard ratio for age $41-50$, and $\geqq 51$ during the 5-year follow-up was $0.94(P=0.70)$ and 0.88 times $(P=0.56)$ lower than in $\leqq 40$ years respectively. The positive association between survival and physician caseload remained statistically significant after multivariate analysis. Patients treated by high-volume physicians had better survival rates (hazard ratio $[\mathrm{HR}]=0.69 ; 95 \%$ confidence interval [CI], 0.50-0.97; $P=0.03$ ), after adjusting for other factors.

\section{Analysis using propensity scores}

Patients were stratified by propensity score and the effect of physician caseload on survival was assessed. The population was stratified into propensity quintiles, as 
previously described. Table 4 shows the survival rates for caseload groups after stratification. The percentage of patients treated by low-volume physicians decreased from the first propensity quintile to the fifth, as predicted by the propensity model. In each of the five strata, patients treated by high-volume physicians had a higher 5-year survival rate. While controlling for propensity score (with fewer patients dying who were treated by high/medium-volume physicians), the $P$ value equaled 0.04 using Cochran-Mantel-Haenszel statistics. This analysis demonstrated a significant difference in survival between patients treated by low vs. high/medium-volume physicians, (adjusted odds ratio $=0.71,95 \%$ CI, 0.51-0.99). The adjusted 5-year survival rates for low vs. high/mediumvolume physicians were $54 \%$ vs. $60 \%$, respectively $(P=$ 0.04).

In summary, cervical cancer patients treated by higher volume physicians showed improved survival. The robustness of this result was demonstrated by two different multivariate analyses, the Cox proportional regression model and stratification by propensity score.

\section{Discussion}

Improved patient outcomes have been correlated with higher caseload volumes. However, there is limited data about the use of brachytherapy in cervical cancer patients. Although the Royal College of Radiologists has made the pragmatic decision to maintain sufficient experience and expertise, they are not backed by any published evidence [16]. We used a Cox proportional hazards model and propensity score to evaluate the relative patient benefit of treatment by high/medium-volume physicians vs. low -volume physicians using cervical cancer brachytherapy. From these results of both forms of multivariate analyses, we found that the 5-year survival rates for brachytherapy patients treated by high/medium -volume physicians were significantly better compared to patients treated by low-volume physicians.
The quality of the risk-adjustment techniques used in analyzing administrative information is an important issue. In the first part of this study, a Cox proportional hazard model was used to compare the effects of high/medium volume versus low volume on survival rates. We found that treatment by high/medium-volume physicians was significantly associated with a lower adjusted hazard ratio for death. Patients treated by high-volume physicians were found to have a $31 \%$ lower risk of death after adjusting for comorbidities and other confounding factors. However, there were differences in clinical conditions between caseload groups. In the second part of our series, propensity score was used to stratify patients into five strata with similar propensity score in order to reduce the effect of selection bias on caseload groups $[14,15,17]$. Patients treated by high/medium-volume physicians were found to have a $6 \%$ relative improvement in adjusted 5-year survival rate $(P=0.04)$ compared to those treated by low-volume physicians.

Several hypotheses have been proposed regarding the relationship between caseload volume and outcome. They suggest that increased caseload may help physicians or hospital staff improve their ability to perform treatment procedures, such as planning and manipulation of the radioactive source to target treatment sites, gauze packing, dose calculation or computerized planning. Careful manipulation of the target volume is important for treatment of cervical cancer with brachytherapy. A team that is comfortable with a higher caseload volume may be more adept at administering radiation dosage which improves locoregional control of cancer and reduces the risk of toxicity to nearby normal organs and tissues.

Although our study revealed some issues that may be useful for policy makers, further research is necessary to identify differences in care and treatment strategies among low-, medium-, and high-volume physicians. In our study, nearly $33 \%$ of patients were treated by only five high-volume radiation oncologists. The viewpoints

Table 4 5-year survival rates of cervical patients according to propensity score strata; low-volume vs. high/mediumvolume physicians ${ }^{a}$

\begin{tabular}{|c|c|c|c|c|c|c|c|}
\hline \multirow[t]{2}{*}{ Propensity score stratum } & \multicolumn{3}{|c|}{ Low-volume physician group } & \multicolumn{3}{|c|}{ High/medium-volume physician group } & \multirow[b]{2}{*}{$P$-value } \\
\hline & No. & $\%$ of stratum & Survival rate (\%) & No. & $\%$ of stratum & Survival rate $(\%)$ & \\
\hline 1 & 112 & 68 & 50 & 51 & 31 & 52 & 0.07 \\
\hline 2 & 84 & 51 & 50 & 80 & 48 & 62 & 0.64 \\
\hline 3 & 50 & 30 & 42 & 114 & 69 & 60 & 0.41 \\
\hline 4 & 19 & 11 & 68 & 145 & 88 & 59 & 0.44 \\
\hline 5 & 15 & 9 & 60 & 148 & 90 & 66 & 0.02 \\
\hline \multirow[t]{2}{*}{ Total } & 280 & & 54 & 538 & & 60 & 0.09 \\
\hline & & & & & & & $0.04^{b}$ \\
\hline
\end{tabular}


of high-volume physicians may influence the development of effective protocols and clinical practice guidelines. Furthermore, the treatment strategies of high-volume physicians should be analyzed and adopted, throughout the country, to improve survival rates.

Our study had several limitations. First, we could not assess the relationship of caseload to stage, tumor size or local control rate because this information was not available from the database. Although this limitation may influence our results, Begg et al., using a SEER-Medicare linked database, reported that cancer stage and patient age were independent of caseload volume [18]. Though the health system in Taiwan is not complete the same as the one in USA, patient in Taiwan are also free to choose any physician no matter the disease severity, stage or comorbidity. In addition, selective referral bias must also be considered, i.e., healthier patients or patients with early-stage disease may tend to be referred to the high-volume physicians. Although healthier patients tend to be wealthier and they advocate for themselves, the National Health Insurance covered approximately 97 percent of the island's population and the hospitals are dispersion in Taiwan. The probability for patients' choice is average. Second, the database does not contain information regarding tobacco use, dietary habits, and body mass index, which might be risk factors for cervical cancer. Taken together, given the robustness of both the evidence and statistical analyses used in this study, these limitations are unlikely to have compromised our results.

In summary, using analyses based on a Cox proportional hazard model and propensity score, we found an association between higher caseload volume and improved survival in cervical cancer patients treated with brachytherapy using population-based data. In conclusion, higher provider caseload affects survival in cervical cancer patients treated with brachytherapy.

\section{Competing interests}

The authors declare that they have no competing interests.

\section{Authors' contributions}

LMS, TSJ, LHY and HSK developed the ideas for these studies, performed much of the work, and drafted the manuscript. LCC, SYC, CWY and LHY designed the study, managed and interpreted the data. LCC performed the statistical analysis. All authors read and approved the final manuscript.

\footnotetext{
Acknowledgments

This study is based in part on data from the National Health Insurance Research Database provided by the Bureau of National Health Insurance, Department of Health and managed by the National Health Research Institutes (Registry number 99029). The interpretation and conclusions contained herein do not represent those of the Bureau of National Health Insurance, Department of Health, or National Health Research Institutes.
}

\section{Author details}

'Department of Radiation Oncology, Buddhist Dalin Tzu Chi Hospital, 2, Ming Sheng Road, Dalin, Chiayi, Taiwan. ${ }^{2}$ Department of Otolaryngology, Buddhist Dalin Tzu Chi Hospital, Chiayi, Taiwan. ${ }^{3}$ Department of Hematology Oncology, Buddhist Dalin Tzu Chi Hospital, Chiayi, Taiwan. ${ }^{4}$ School of Medicine, Tzu Chi University, Hualien, Taiwan.
Received: 13 October 2013 Accepted: 10 October 2014

Published online: 25 October 2014

\section{References}

1. Chang CM, Huang KY, Hsu TW, Su YC, Yang WZ, Chen TC, Chou P, Lee CC: Multivariate analyses to assess the effects of surgeon and hospital volume on cancer survival rates: a nationwide population-based study in Taiwan. PLoS One 2012, 7:e40590.

2. Lee CC, Huang TT, Lee MS, Su YC, Chou P, Hsiao SH, Chiou WY, Lin HY, Chien SH, Hung SK: Survival rate in nasopharyngeal carcinoma improved by high caseload volume: a nationwide population-based study in Taiwan. Radiat Oncol 2011, 6:92.

3. Peters WA 3rd, Liu PY, Barrett RJ 2nd, Stock RJ, Monk BJ, Berek JS, Souhami L, Grigsby P, Gordon W Jr, Alberts DS: Concurrent chemotherapy and pelvic radiation therapy compared with pelvic radiation therapy alone as adjuvant therapy after radical surgery in high-risk early-stage cancer of the cervix. J Clin Oncol 2000, 18:1606-1613.

4. Ferrigno R, dos Santos Novaes PE, Pellizzon AC, Maia MA, Fogarolli RC, Gentil AC, Salvajoli JV: High-dose-rate brachytherapy in the treatment of uterine cervix cancer. Analysis of dose effectiveness and late complications. Int J Radiat Oncol Biol Phys 2001, 50:1123-1135.

5. Lin CC, Lin HC: Effects of surgeon and hospital volume on 5-year survival rates following oral cancer resections: the experience of an Asian country. Surgery 2008, 143:343-351.

6. Peltoniemi P, Huhtala H, Holli K, Pylkkanen L: Effect of surgeon's caseload on the quality of surgery and breast cancer recurrence. Breast 2012, 21:539-543.

7. Markar SR, Karthikesalingam A, Thrumurthy S, Low DE: Volume-outcome relationship in surgery for esophageal malignancy: systematic review and meta-analysis 2000-2011. J Gastrointest Surg 2012, 16:1055-1063.

8. Goodney PP, Stukel TA, Lucas FL, Finlayson EV, Birkmeyer JD: Hospital volume, length of stay, and readmission rates in high-risk surgery. Ann Surg 2003, 238:161-167.

9. Deyo RA, Cherkin DC, Ciol MA: Adapting a clinical comorbidity index for use with ICD-9-CM administrative databases. J Clin Epidemiol 1992, 45:613-619.

10. Braaten $T$, Weiderpass $E$, Lund $E$ : Socioeconomic differences in cancer survival: the Norwegian women and cancer study. BMC Public Health 2009, 9:178.

11. Kwok J, Langevin SM, Argiris A, Grandis JR, Gooding WE, Taioli E: The impact of health insurance status on the survival of patients with head and neck cancer. Cancer 2010, 116:476-485.

12. Chen CY, Liu CY, Su WC, Huang SL, Lin KM: Factors associated with the diagnosis of neurodevelopmental disorders: a population-based longitudinal study. Pediatrics 2007, 119:e435-e443.

13. Joffe MM, Rosenbaum PR: Invited commentary: propensity scores. Am J Epidemiol 1999, 150:327-333.

14. Rubin DB: Tasks in statistical inference for studying variation in medicine. Med Care 1993, 31:YS103-YS110.

15. Rubin DB: Estimating causal effects from large data sets using propensity scores. Ann Intern Med 1997, 127:757-763.

16. Symonds P, Davidson S, Vale C, Drinkwater K: Caseload and outcome after brachytherapy. Clin Oncol 2013, 25:519-521.

17. D'Agostino RB Jr: Propensity score methods for bias reduction in the comparison of a treatment to a non-randomized control group. Stat Med 1998, 17:2265-2281.

18. Begg CB, Cramer LD, Hoskins WJ, Brennan MF: Impact of hospital volume on operative mortality for major cancer surgery. JAMA 1998, 280:1747-1751.

doi:10.1186/s13014-014-0234-2

Cite this article as: Lee et al: Higher caseload improves cervical cancer survival in patients treated with brachytherapy. Radiation Oncology 2014 9:234. 